“( 2016 IEEE. Personal use of this material is permitted. Permission from IEEE must be obtained for all other uses, in any current or future media, including

reprinting/republishing this material for advertising or promotional purposes, creating new collective works, for resale or redistribution to servers or lists, or reuse of any copyrighted component of this work in other works." 


\title{
On the Usage of Geolocation Aware Spectrum Measurements for Incumbent Location and Transmit Power Detection
}

\author{
Beeshanga Abewardana Jayawickrama, Member, IEEE, Eryk Dutkiewicz, Member, IEEE, \\ Markus Mueck, Member, IEEE, and Ying He, Member, IEEE
}

\begin{abstract}
Determining the geographical area that needs to be excluded due to incumbent activity is critical to realise high spectral utilisation in spectrum sharing networks. This can be achieved by estimating the incumbent location and transmit power. However keeping the hardware complexity of sensing nodes to a minimum and scalability are critical for spectrum sharing applications with a commercial intent. We present a discrete space $\ell_{1}$-norm minimisation solution based on geolocation aware energy detection measurements. In practice the accuracy of geolocation tagging is limited. We capture the impact as a basis mismatch and derive the necessary condition that needs to be satisfied for successful detection of multiple incumbents' location and transmit power. We find the upper bound for the probability of eliminating the impact of limited geolocation tagging accuracy in a lognormal shadow fading environment, which is applicable to all generic $\ell_{1}$-norm minimisation techniques. We propose an algorithm based on Orthogonal Matching Pursuit that decreases the residual in each iteration by allowing a selected set of basis vectors to rotate in a controlled manner. Numerical evaluation of the proposed algorithm in a Licensed Shared Access network shows a significant improvement in the probability of Missed Detection and False Alarm.
\end{abstract}

Index Terms-Sparse matrices, Spatial correlation, Cognitive radio, Orthogonal Matching Pursuit, Licensed Shared Access.

\section{INTRODUCTION}

The series of wireless and in particular cellular technology generations manifested over the last few decades have been a great success providing much improved throughput and quality of service in each generation. Although the engineered solutions have well defined limits, the ever growing demand for traffic is limited only by the users' imagination. There was a time that spectrum refarming was an option, however it is becoming increasingly difficult to refarm the spectrum below $6 \mathrm{GHz}$.

The Dynamic Spectrum Access concept has been proposed in literature as a spectrum sharing approach that increases the efficiency in spectrum usage. In such systems there are

Copyright (c) 2015 IEEE. Personal use of this material is permitted. However, permission to use this material for any other purposes must be obtained from the IEEE by sending a request to pubs-permissions@ieee.org.

This work was supported in part by the Intel Corporation University Research Office.

B. A. Jayawickrama and E. Dutkiewicz are with School of Computing and Communications, University of Technology Sydney, Sydney, Australia (e-mail: beeshanga.jayawickrama@uts.edu.au; eryk.dutkiewicz@uts.edu.au).

M. Mueck is with Intel Mobile Communications, Munich, Germany (email: markus.dominik.mueck@intel.com).

Y. He is with Department of Engineering, Macquarie University, Sydney, Australia (e-mail: y.he@mq.edu.au) two levels of users - incumbents that have the privilege to use the spectrum at their discretion, and secondary users that can use the spectrum with no interruption to incumbents. The concept has been backed by a vast number of research work in spectrum sensing for detecting whether an incumbent is active [1]-[4]. Different spectrum sensing approaches such as Energy Detection, Matched Filter Detection and Cyclostationary Detection have been thoroughly investigated in literature, in frequency-time domain.

Although the spectrum sharing concept emerged over a decade ago, a commercially viable system is still overdue. To promote efficient use of spectrum regulatory bodies are now promoting spectrum sharing. In Europe, European Telecommunications Standards Institute Reconfigurable Radio Systems (ETSI RRS) Technical Standardisation Committee is developing a two tier spectrum sharing framework named Licensed Shared Access (LSA) for the 2300-2400 MHz band [5]. In the initial stage the LSA sharing time scale is in the order of years/months, which is expected to reduce down to a minutes/seconds scale only. The development of LSA is currently at a mature stage, standardisation activities are expected to be completed in 2015. Also it should be noticed that in the USA, Federal Communications Commission (FCC) is beginning to lay out the rules and regulations for a 3 tier spectrum sharing licensing model in the $3550-3650 \mathrm{MHz}$ band [6].

A common attribute of recent developments in spectrum sharing is that incumbents communicate with secondary spectrum users. Particularly in the LSA framework, incumbents inform the active time of the day, frequency and an approximate geographical region where the spectrum is already being used. The incumbent is able to provide highly accurate information on the active time of the day and frequency. However it can only predict the active geographical area with limited accuracy. Even though an incumbent is actively using the spectrum in a large region, there will be local areas where incumbent received power is too low. In such areas the secondary users should be able to utilise the spectrum. Therefore the traditional frequency-time domain based spectrum sensing approaches that focus on millisecond scale spectrum sharing do not augment well with emerging standardisation activities. Hence, spectrum sensing should rather be focused on finding the area where the incumbent is active in the spatial domain on a minutes/seconds time scale.

Literature proposes to use geolocation tagged measurements 
in this regard [7]-[13]. In [7], the authors emphasise the ability to detect more spectrum opportunities through location awareness. In [14], the authors find an optimal detection threshold that minimised a utility function of the hidden node probability. In [8]-[10], the authors propose a concept named Radio Environment Map (REM) to enhance contextual awareness of wireless networks in spatial domain. REM can be envisioned as a heatmap that shows the propagation pattern of a RF signal in space. A REM of the incumbent signal clearly shows spectrum holes in space.

When finding the geographical area where the incumbents are active, the incumbent location, transmit power and the radiation pattern of the incumbent antenna are critical parameters. The use cases of LSA suggest the incumbents tend to have an omnidirectional antennas [15]. Therefore active area is a function of the incumbent location and the transmit power. Following this notion, in [16], [17], the authors present a framework which can be used to locate an incumbent. However the feasibility of this framework in the presence of multiple incumbents has not been reported. A scalable approach that can jointly detect the transmit power and the location of multiple incumbents through a reasonable number of measurements from less complex measurement devices is preferred for commercial applications.

Therefore, in our previous work [11], [12], we formulated incumbent transmit power and location detection as a discrete space $\ell_{1}$-norm minimisation problem. We showed that it is possible to detect the locations and transmit powers of multiple incumbents by exploiting the sparsity in spatial domain. However we contemplated that all nodes collecting measurements are located on a subset of predetermined locations (we refer to this scenario as on-grid). The channel between any predetermined location to other predetermined locations was estimated through training.

In applications with a commercial intent (such as LSA), low cost measurement devices may not be located in predetermined points in space as such (we refer to this scenario as offgrid). When measurement devices are off-grid, the channel from a predetermined location to a device is not known. The $\ell_{1}$-norm minimisation techniques suggested in [11], [12] are less effective in such an environment, as the basis vectors become erroneous. In this paper we extend our previous work by addressing the issue of joint detection of incumbent location and transmit power through geolocation aware measurements from a network of off-grid measurement devices.

As we suggested the harmful effect of off-grid measurement devices can be mitigated by infinitely increasing the resolution of the predetermined reference point layout [11]. Another possible solution is to estimate the channel to each off-grid measurement device through training. Further, the literature suggests using the well-known Dictionary Learning techniques [18]-[20] to estimate the basis vectors. Although such approaches produce more accurate estimations for basis vectors in $\ell_{1}$-norm minimisation, they result in increased traffic overhead.

With a strong emphasis on not increasing the traffic overhead any further, we consider possible signal processing techniques. We use the channel estimations between predetermined reference points as the $\ell_{1}$-norm basis. However we capture the off-grid error as an unknown rotation/scale of the known basis vectors. We propose to allow basis vectors to rotate in a controlled manner within well defined bounds to mitigate the off-grid errors. Our approach significantly reduces the off-grid error while strictly maintaining the same traffic overhead as our previous work.

Main contributions of this paper are as follows:

- Summarise the incumbent location and transmit power detection as a discrete space $\ell_{1}$-norm minimisation problem based on our previous work [11], [12]

- When using $\ell_{1}$-norm minimisation, the dictionary matrix should satisfy the Restricted Isometry Property (RIP). Although the previous numerical results indicated the dictionary matrix meets the RIP requirement, no analytical proof was found in literature. This paper proves the dictionary matrix meets the RIP condition.

- Our main contribution of this paper is extending the discrete space model by taking into account off-grid Measurement Capable Devices (MCDs). The impact of off-grid nodes is captured as a mismatch in the dictionary matrix. Majority of the literature suggests using Dictionary Learning techniques to discover the dictionary matrix [18]-[20]. However, in this paper we build on the recent work in [21], [22]. Our approach falls into the class of Orthogonal Matching Pursuit (OMP) algorithms, and it reduces the impact of off-grid nodes without incurring any additional communication overhead. This is achieved by allowing the dictionary vectors to rotate in the direction of the residual at each iteration. Further, we find the necessary condition that needs to be satisfied in order to overcome the harmful impact of the off-grid MCDs.

- We apply the proposed improvement to a cellular network operating in the $2300-2400 \mathrm{MHz}$ band under a LSA agreement. The LSA licensee network uses geolocation tagged measurements from mobile devices and basestations to determine the incumbent location and transmit power.

- Numerical results show a substantial improvement in the ability to determine the location and transmit power of sparsely distributed incumbents. The approach in [21] tends to overestimate the number of incumbents while [22] underestimates. The proposed improvement gets significantly closer to the actual number of incumbents. Additionally, a clear improvement is evident in the probability of False Alarm (FA) and Missed Detection (MD).

In Section II, we describe the system model assumed in our work. In Section III, we formulate the joint incumbent transmit power and location detection problem as an $\ell_{1}$-norm minimisation problem. We also prove that Restricted Isometry Property holds in this scenario. In Section IV, we find the upper bound of the probability of successful detection that can be achieved by any $\ell_{1}$-norm minimisation technique, when using off-grid measurements. We also develop a reconstruction algorithm to mitigate the harmful impact of off-grid measurements by allowing basis vectors to rotate within well defined bounds. 


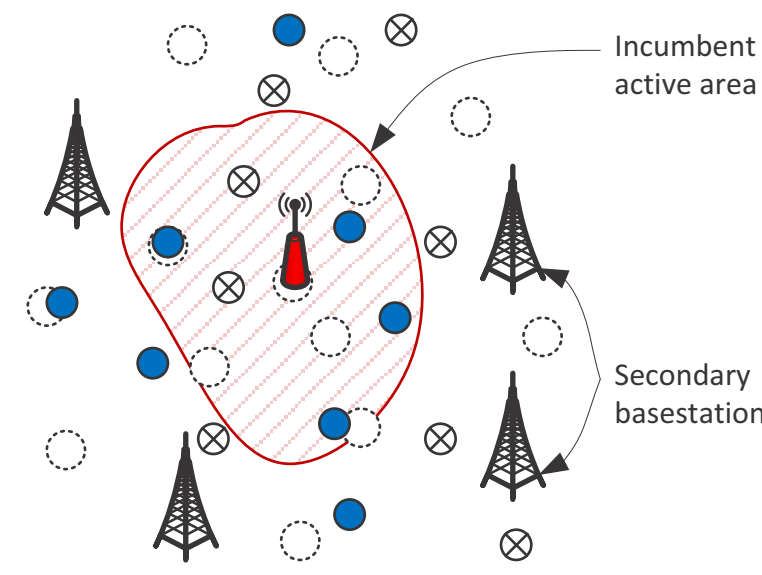

MCD - taking

measurements

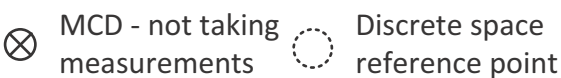

Fig. 1: System model. Single incumbent scenario is illustrated here, although this work considers the multiplicity of incumbents. Incumbents are considered to be on any subset of reference points, Measurement Capable Devices (MCDs) could be anywhere in space.

In Section V, we summarise the numerical results obtained by simulating the proposed approach in a LSA network operating in 2300-2400 MHz band.

\section{SySTEM MODEL}

Consider a set of discrete $N_{p}$ points in two dimensional space covering a region where incumbents could be active. We allow $k$ incumbents to actively transmit in a subset of $k$ sparsely distributed discrete reference points. Among all Measurement Capable Devices (MCDs) in the LSA network $N_{s}$ MCDs are collecting geolocation tagged Received Signal Strength (RSS) measurements of the incumbent signal. A MCD could be any transceiver in the LSA network (a mobile device or a basestation) and/or dedicated sensor nodes with self geolocalisation ability. Geolocation tagged RSS measurements are then processed in a central location to determine at which reference points the incumbents are active at, and the transmit power. The system model is shown in Fig. 1.

Let $r_{i}(t)$ be the received signal at any $i$ th reference point at time $t$, that can be written as,

$$
r_{i}(t)=\sum_{j=1}^{N_{p}} h_{i j}(t) s_{j}(t)+n_{i}(t)
$$

where $h_{i j}$ is the complex channel gain (i.e. transfer function) from the $j$ th transmit reference point to the $i$ th receive reference point. The transmit signal at the $j$ th reference point is denoted by $s_{j}$, if no transmission occurs at the $j$ th reference point $s_{j}=0$ condition is held. $n_{i}$ is the Additive White Gaussian Noise (AWGN). We assume a narrow band and frequency-flat fading channel.

Hence, the received signal at all grid points can be written in vector notation as,

$$
r(t)=\left(r_{1}(t), r_{2}(t), \ldots, r_{N_{p}}(t)\right)^{T}=H(t) s(t)+n(t)
$$

where $n(t)$ is a $N_{p} \times 1$ AWGN vector, $s(t)=$ $\left(s_{1}(t), s_{2}(t), \ldots, s_{N_{p}}(t)\right)^{T}$ and $(\cdot)^{T}$ is the transpose of $(\cdot)$. Further $H$ is a $N_{p} \times N_{p}$ matrix (not $N_{s} \times N_{p}$ ) as follows,

$$
H=\left(\begin{array}{cccc}
h_{11} & h_{12} & \cdots & h_{1 N_{p}} \\
h_{21} & h_{22} & \cdots & h_{2 N_{p}} \\
\vdots & \vdots & \ddots & \vdots \\
h_{N_{p} 1} & h_{N_{p} 2} & \cdots & h_{N_{p} N_{p}}
\end{array}\right)
$$

The instantaneous Received Signal Strength (RSS) at discrete time sample $t$ can be calculated as $r(t) \circ r(t)^{*}$, where $\circ$ is the Hadamard product. The mean value calculated over $M$ samples at the MCDs can be written as,

$$
P_{r}(t)=\frac{1}{M} \sum_{i=0}^{M-1} r(t-i) \circ r(t-i)^{*}
$$

We assume a signal transmitted from any $i$ th point is uncorrelated with a signal transmitted at $j$ th point. Additionally we also assume that noise is spatio-temporally white and independent of transmitted signal. Therefore we can rewrite (4) as follows,

$$
P_{r}(t)=\Psi(t) P_{t}(t)+\sigma_{n}^{2} \mathbb{1}_{N_{p}}
$$

where $\Psi(t)$ is the $N_{p} \times N_{p}$ channel gain matrix given by $[\Psi]_{i j}(t)=\frac{1}{M} \sum_{i=0}^{M-1}\left[\left|h_{i j}(t-i)\right|^{2}\right], P_{t}$ is the $N_{p} \times 1$ transmit power vector given by $\left[P_{t}\right]_{i}=\frac{1}{M} \sum_{i=0}^{M-1}\left[\left|s_{i}\right|^{2}\right], \sigma_{n}^{2}$ is the variance of AWGN and $\mathbb{1}_{N_{p}}$ is a $N_{p} \times 1$ vector of ones. We consider that $N_{s}$ MCDs are performing spectrum measurements at any given time. Hence the RSS at $N_{s}$ reference points nearest to that subset of MCDs taking measurements can be written as $P_{s}\left(\subset P_{r}\right)$,

$$
P_{s}(t)=\Phi(t) \Psi(t) P_{t}(t)+\sigma_{n}^{2} \mathbb{1}_{N_{s}}
$$

where $\Phi(t)$ is the $N_{s} \times N_{p}$ matrix representing the geolocation of spectrum sensing nodes as follows,

$$
[\Phi]_{k j}= \begin{cases}1 & \text { if } k^{\text {th }} \mathrm{MCD} \text { is at } j^{\text {th }} \text { reference point } \\ 0 & \text { otherwise }\end{cases}
$$

The geo-location of the sensing nodes is assumed to have been provided by an external service such as the Global Positioning System (GPS). Note that the impact of MCDs being off-grid is analysed in Section IV.

The incumbent location and transmit power detection problem can be stated as solving the underdetermined system in (6) for $P_{t}$. The indices of non-zero elements in $P_{t}$ vector correspond to incumbent locations, whereas the values at those indices are the transmit powers of each incumbent.

\section{DETERMINING THE INCUMBENT LOCATIONS AND TRANSMIT POWERS}

In a typical operating environment of a secondary system, multiple incumbents that are sparsely distributed in spatial domain might be present. Therefore, the large fraction of elements in $P_{t}(t)$ vector will be zero, while the non-zero elements will be sparse. Motivated by this characteristic, we investigate the possibility of using $\ell_{l}$-norm minimisation techniques to solve the underdetermined problem in (6). However, when 
using $\ell_{1}$-norm minimisation, one must ensure the dictionary matrix $\Phi(t) \Psi(t)$ satisfies the Restricted Isometry Property (RIP) for the intended application. In Theorem 1 we prove $\Phi(t) \Psi(t)$ follows RIP, the rest of the section formulates a $\ell_{1}$ norm minimisation problem.

Any element in $\Psi(t)$ can be written as $[\Psi]_{i j}(t)=$ $\frac{1}{M} \sum_{i=0}^{M-1}\left[\left|h_{i j}(t-i)\right|^{2}\right]$. In the most dynamic form, spectrum sharing related standardisation activities are focusing on sharing in a seconds scale as discussed in Section I. Therefore, the effect of fast fading can be considered to have been averaged out over $M$ samples. Hence, any element in $\Psi(t)$ can be modelled as,

$$
[\Psi]_{i j}(t)=\frac{1}{M} \sum_{i=0}^{M-1}\left[\left|h_{i j}(t-i)\right|^{2}\right] \sim G_{i j}(t) L_{i j}(t)
$$

where $G_{i j}$ is a coefficient capturing large scale shadow fading and $L_{i j}$ captures the pathloss. In the rest of the paper we omit the time $(t)$ notation for simplicity in illustration.

Theorem 1: Let the number of incumbents be $k$. When the number of MCDs $N_{s} \geq c_{1} k \log \left(N_{p} / k\right)$ where $c_{1}$ is a constant, the probability that $\Phi \Psi$ satisfies

$$
1-\delta_{k} \leq \frac{\|\Phi \Psi v\|_{2}^{2}}{\|v\|_{2}^{2}} \leq 1+\delta_{k}
$$

for some $\delta_{k} \in(0,1)$ and any $k$-sparse vector $v$ tends to 1 . In other words, the $\Phi \Psi$ matrix satisfies the Restricted Isometry Property (RIP).

Proof: The proof is provided in Appendix A

In any incumbent wireless network, it is reasonable to assume the transmitters are sparsely distributed. Hence, we assume that $P_{t}$ is a sparse vector. Computing $\ell_{0}$-norm essentially counts the number of non-zero elements in $P_{t}$. Since $\Phi \Psi$ satisfies RIP, we formulate the $\ell_{0}$ minimisation problem as follows,

$$
\begin{gathered}
\underset{P_{t}^{*}}{\arg \min }\left\|P_{t}^{*}\right\|_{0} \\
\text { subject to: }\left\|P_{s}-\sigma_{n}^{2} \mathbb{1}_{N_{s}}-\Phi \Psi P_{t}^{*}\right\|_{2} \leq \hat{\vartheta}
\end{gathered}
$$

where $\|(\cdot)\|_{p}$ is the $\ell_{p}$ norm and $\hat{\vartheta}$ is a constraint relaxation parameter. However this problem is numerically unstable due to non-convexity [23]. Therefore we convert the $\ell_{0}$ minimisation to a $\ell_{1}$ minimisation problem as follows,

$$
\begin{gathered}
\underset{P_{t}^{*}}{\arg \min }\left\|P_{t}^{*}\right\|_{1} \\
\text { subject to: }\left\|P_{s}-\sigma_{n}^{2} \mathbb{1}_{N_{s}}-\Phi \Psi P_{t}^{*}\right\|_{2} \leq \vartheta
\end{gathered}
$$

Please note that constraint relaxation parameter changes when converting (10) into the $\ell_{1}$-norm minimisation form in (11). The $\ell_{1}$ minimisation problem in (11) can be solved in polynomial time using a log barrier algorithm [24]. Since $\Psi$ is however a large and dense matrix, literature suggests using greedy algorithms such as Orthogonal Matching Pursuit (OMP) for lower computational complexity [21]. The primary advantage of the $\ell_{1}$ minimisation technique is the robustness even in the presence of multiple incumbents. However, the accuracy of $P_{t}$ estimation is dependant on the $\Phi \Psi$ estimation.

\section{IMPACT OF OFF-GRID MEASUREMENT CAPABLE DEVICES}

A common drawback in any discrete system is the quantization error. In a typical operating environment, any transceiver with self-geolocalisation capability in the secondary system could be considered as a MCD. Since the MCDs may not be located exactly on the set of $N_{p}$ reference points, the channel from the $j$ th reference point to $i$ th MCD will be different from $[\Psi]_{i j}$. One approach would be to increase the resolution of the reference point layout until all $N_{s}$ nodes are on-grid. This will increase the size of $\Phi$ and $\Psi$ matrices resulting in too many possible solutions for the underdetermined problem in (11).

Therefore, we propose an alternative approach to capture the off-grid error (spatial domain quantisation error). The $N_{s}$ MCDs taking measurements in continuous two dimensional space are related to the nearest $N_{s}$ reference points. The spectrum measurements taken by the MCDs can be written similar to $(6)$, where $[\tilde{\Psi}]_{i j}(t)=\frac{1}{M} \sum_{i=0}^{M-1}\left[\left|\tilde{h}_{i j}(t-i)\right|^{2}\right]$ is the channel from $j$ th grid point to $i$ th MCD. Hence the measurement process becomes,

$$
\tilde{P}_{s}=\Phi \tilde{\Psi} P_{t}+\sigma_{n}^{2} \mathbb{1}_{N_{s}}
$$

Reconstruction process should have been,

$$
\begin{gathered}
\underset{P_{t}^{*}}{\arg \min }\left\|P_{t}^{*}\right\|_{1} \\
\text { subject to: }\left\|P_{s}-\sigma_{n}^{2} \mathbb{1}_{N_{s}}-\Phi \tilde{\Psi} P_{t}^{*}\right\|_{2} \leq \vartheta
\end{gathered}
$$

Please note that (13) uses $\tilde{\Psi}$, whereas (10) and (11) use $\Psi$ as the channel matrix. However, the channel from any selected reference point to the $i$ th MCD is not known, unless the $i$ th MCD is exactly at the $i$ th grid point. Therefore the reconstruction has to be done with (11) which introduces an error in the reconstruction process. In this section we find the necessary condition that needs to be satisfied to overcome this mismatch and propose an algorithm to minimise the error caused.

\section{A. Necessary condition for recovery}

Proposition 1: In a quasi dynamic spectrum access scenario (i.e. time samples average out the small scale fading effect), the shadow fading coefficient from the $j$ th grid point to $i$ th MCD $\tilde{G}_{i j}$ is given by,

$$
\left[\tilde{G}_{i j}\right]_{\mathrm{dB}}=\rho\left[G_{i j}\right]_{\mathrm{dB}}+\sqrt{1-\rho^{2}} R_{i j}
$$

where $R_{i j} \sim \mathcal{N}\left(0, \sigma_{g}^{2}\right)$ and,

$$
\rho=R(\Delta x)=\exp \left(\frac{-|\Delta x|}{d_{c}}\right)
$$

is the autocorrelation of shadowing when MCD is $|\Delta x|$ away from the nearest grid point [25, Eq. (6)]. The decorrelation distance of shadowing $d_{c}$ is dependent on the environment.

Proof: Large scale shadow fading is known to follow a log-normal distribution with exponentially decaying autocorrelation given by $\rho$ [25], [26]. Therefore the correlation between 
shadow fading coefficient from the $j$ th grid point to the $i$ th grid point and nearest MCD can be written as,

$$
R=\left(\begin{array}{cc}
1 & \exp \left(-|\Delta x| / d_{c}\right) \\
\exp \left(-|\Delta x| / d_{c}\right) & 1
\end{array}\right)
$$

We find a lower triangular matrix $R_{L}$ using Cholesky decomposition such that $R=R_{L} R_{L}^{T}$ holds.

$$
R_{L}=\left(\begin{array}{cc}
1 & 0 \\
\exp \left(-|\Delta x| / d_{c}\right) & \sqrt{1-\exp \left(-2|\Delta x| / d_{c}\right)}
\end{array}\right)
$$

Hence the correlated shadowing coefficients $\left[G_{i j}\right]_{\mathrm{dB}}$ and $\left[\tilde{G}_{i j}\right]_{\mathrm{dB}}$ can be written as,

$$
\left(\begin{array}{c}
{\left[G_{i j}\right]_{\mathrm{dB}}} \\
{\left[\tilde{G}_{i j}\right]_{\mathrm{dB}}}
\end{array}\right)=R_{L}\left(\begin{array}{c}
{\left[G_{i j}\right]_{\mathrm{dB}}} \\
R_{i j}
\end{array}\right)
$$

By substituting $\rho=\exp \left(-|\Delta x| / d_{c}\right)$, we can deduce (14).

Proposition 2: Let the channel from the $j$ th reference point to the $i$ th MCD be $\tilde{\Psi}_{i j}$. Let the channel from the same point to the nearest reference point that corresponds to the $i$ th $\mathrm{MCD}$ be $\Psi_{i j}$. Off-grid perturbations can be modelled as,

$$
\tilde{\Psi}_{i j}=\Psi_{i j} \tilde{\psi}_{i j}
$$

where $\tilde{\psi}_{i j}=10^{\cdot 1\left(\sqrt{1-\rho^{2}} R_{i j}-(1-\rho)\left[G_{i j}\right]_{\mathrm{dB}}\right)}$

Proof: The measurement channel from the $j$ th grid point to the $i$ th node can be written as $\tilde{\Psi}_{i j}=\tilde{L}_{i j} \tilde{G}_{i j}$. Since the $i$ th node is taking measurements in the near neighbourhood of the $i$ th reference point we can assume the pathloss variation is minor.

$$
\tilde{\Psi}_{i j}=\tilde{L}_{i j} \tilde{G}_{i j} \approx L_{i j} \tilde{G}_{i j}
$$

Variation of shadow fading due to off-grid nodes can be modelled as shown in Proposition 1. Clearly it can be shown that,

$$
\tilde{\Psi}_{i j}=L_{i j} G_{i j} \tilde{\psi}_{i j}=\Psi_{i j} \tilde{\psi}_{i j}
$$

Proposition 3: The angle $\varphi$ between any two vectors $\Psi_{j}$ and $\Psi_{k}$ is an acute angle.

Proof: Consider the inner product,

$$
\Psi_{j} \cdot \Psi_{k}=\sum_{i=1}^{N_{p}} \Psi_{i j} \Psi_{i k}=\sqrt{\sum_{i=1}^{N_{p}} \Psi_{i j}} \sqrt{\sum_{i=1}^{N_{p}} \Psi_{i k}} \cos (\varphi)
$$

Since $\Psi_{i j}, \Psi_{i k}>0\left(\forall 1 \leq j, k \leq N_{p}\right)$, clearly $\cos (\varphi)>0$. Therefore $\varphi<\pi / 2$ is an acute angle.

Theorem 2: Consider any column vector $\Psi_{j}$. The error caused by off-grid measurement process could be corrected only if,

$$
\sum_{i=1}^{N_{p}}\left(\Psi_{i j}-\cos (\varphi / 2) \sqrt{\sum_{i=1}^{N_{p}} \Psi_{i j}^{2}}\right) \Psi_{i j} \tilde{\psi}_{i j}>0
$$

condition is met.

Proof: The error caused by the measurement process using $\tilde{\Psi}_{i j}$ channel and estimation using $\Psi_{i j}$ can be modeled as an unknown rotation of the column vector $\Psi_{j}$ by an angle $\vartheta$ and a stretch factor. Possible realisations of $\tilde{\Psi}_{j}$ can be

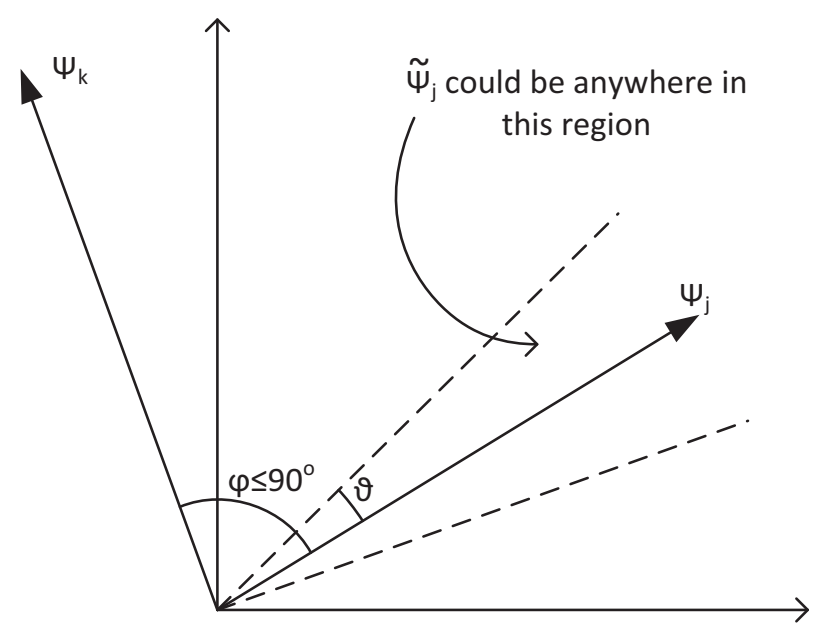

Fig. 2: The error caused by the measurement process can be captured as an unknown rotation and stretch. To recover the signal the unknown rotation should be upper bounded as $\vartheta<$ $\varphi / 2$.

visualised as a $N_{p}$-dimensional cone around the vector $\Psi_{j}$, shown in Fig. 2. Similarly any other vector $\Psi_{k}($ where $k \neq j$ ) will also have a $N_{p}$-dimensional cone around it, within which $\Psi_{k}$ is subject to rotation and scaling. Any overlap between those two spaces will result in a non-unique projection which makes the recovery not possible. Therefore, to avoid any overlap it is necessary to satisfy,

$$
\vartheta<\varphi / 2
$$

where $\varphi$ is the angle between $\Psi_{j}$ and any column vector $\Psi_{k}(k \neq j)$.

From Proposition 3, $\varphi$ is an acute angle. Therefore,

$$
\Leftrightarrow \cos (\vartheta)=\frac{\sum_{i=1}^{N_{p}} \Psi_{i j} \tilde{\Psi}_{i j}}{\sqrt{\sum_{i=1}^{N_{p}} \Psi_{i j}^{2}} \sqrt{\sum_{i=1}^{N_{p}} \tilde{\Psi}_{i j}^{2}}}>\cos (\varphi / 2)
$$

From Proposition 2, and omitting 1 to $N_{p}$ indexing in the summations we write,

$$
\Leftrightarrow \frac{\sum \Psi_{i j}^{2} \tilde{\psi}_{i j}}{\sqrt{\sum \Psi_{i j}^{2}} \sqrt{\sum \Psi_{i j}^{2} \tilde{\psi}_{i j}^{2}}}>\cos (\varphi / 2)
$$

Since $\Psi_{i j} \tilde{\psi}_{i j}>0$ we can write $\sum \Psi_{i j} \tilde{\psi}_{i j}>\sqrt{\sum \Psi_{i j}^{2} \tilde{\psi}_{i j}^{2}}$. Therefore,

$$
\begin{aligned}
& \Leftarrow \frac{\sum \Psi_{i j}^{2} \tilde{\psi}_{i j}}{\sum \Psi_{i j} \tilde{\psi}_{i j}}>\cos (\varphi / 2) \sqrt{\sum \Psi_{i j}^{2}} \\
& \Leftrightarrow \sum\left(\Psi_{i j}-\cos (\varphi / 2) \sqrt{\sum \Psi_{i j}^{2}}\right) \Psi_{i j} \tilde{\psi}_{i j}>0
\end{aligned}
$$

Proposition 4: In each vector $\Psi_{j}$, there exists at most one element $\Psi_{i j}$ which satisfies $\Psi_{i j}>\cos (\varphi / 2) \sqrt{\sum \Psi_{i j}^{2}}$. 
Proof: A proof by contradiction is provided for this proposition. Consider a $3 \times 1$ vector $\Psi_{j}=\left(\Psi_{1 j}, \Psi_{2 j}, \Psi_{3 j}\right)^{T}$. Let,

$$
\Psi_{1 j}, \Psi_{2 j}>\cos (\varphi / 2) \sqrt{\Psi_{1 j}^{2}+\Psi_{2 j}^{2}+\Psi_{3 j}^{2}}
$$

Since $\Psi_{i j}>0$ and $\varphi$ is an acute angle we write,

$$
\begin{aligned}
& \Rightarrow \Psi_{1 j}>\cos (\varphi / 2) \sqrt{\Psi_{1 j}^{2}+\Psi_{2 j}^{2}+\Psi_{3 j}^{2}} \\
& \Leftrightarrow \Psi_{1 j}^{2}>\cos ^{2}(\varphi / 2)\left(\Psi_{1 j}^{2}+\Psi_{2 j}^{2}+\Psi_{3 j}^{2}\right) \\
& \Leftrightarrow \Psi_{1 j}^{2}>\frac{1}{\tan ^{2}(\varphi / 2)}\left(\Psi_{2 j}^{2}+\Psi_{3 j}^{2}\right)
\end{aligned}
$$

For an acute angle $\varphi$, we can write $\tan ^{2}(\varphi / 2) \leq 1$. Therefore,

$$
\begin{aligned}
& \Rightarrow \Psi_{1 j}^{2}>\Psi_{2 j}^{2}+\Psi_{3 j}^{2} \\
& \Rightarrow \Psi_{1 j}^{2}>\Psi_{2 j}^{2}
\end{aligned}
$$

Similarly, considering $\Psi_{2 j}$ we get,

$$
\Psi_{2 j}^{2}>\Psi_{1 j}^{2}
$$

which is a contradiction. Hence, there exists at most one element such that $\Psi_{i j}>\cos (\varphi / 2) \sqrt{\sum \Psi_{i j}^{2}}$.

Theorem 3: The probability of successful recovery of $P_{t}$ using measurements from off-grid MCDs is upper bounded by,

$$
1-\prod_{i=1}^{N_{p} \backslash\{k\}} Q\left(\frac{(1-\rho)\left[G_{i j}\right]_{\mathrm{dB}}+\left[f_{i j}\right]_{\mathrm{dB}}}{\sigma_{g}^{2} \sqrt{1-\rho^{2}}}\right)
$$

where,

$$
f_{i j}=\frac{\Omega_{k}}{N_{p}-1} \frac{1}{\left(\cos (\varphi / 2) \sqrt{\sum \Psi_{i j}^{2}}-\Psi_{i j}\right) \Psi_{i j}}
$$

Proof: Let $\Omega_{k}=\left(\Psi_{k j}-\cos (\varphi / 2) \sqrt{\sum \Psi_{i j}^{2}}\right) \Psi_{k j} \tilde{\psi}_{k j}$. The $k$ th element of $\Psi_{j}$ is $\Psi_{k j}$. From Proposition 4, consider the scenario where $\Omega_{k}>0$ exists for exactly one value of $1 \leq k \leq N_{p}$. From Theorem 2, the error caused by off-grid nodes can be eliminated only if,

$$
\Omega_{k}-\sum_{i=1}^{N_{p} \backslash\{k\}}\left(\cos (\varphi / 2) \sqrt{\sum \Psi_{i j}^{2}}-\Psi_{i j}\right) \Psi_{i j} \tilde{\psi}_{i j}>0
$$

Clearly this condition will not be satisfied when,

$$
\left(\cos (\varphi / 2) \sqrt{\sum \Psi_{i j}^{2}}-\Psi_{i j}\right) \Psi_{i j} \tilde{\psi}_{i j}>\frac{\Omega_{k}}{N_{p}-1}
$$

for each $i \in\left\{1, \ldots, N_{p}\right\} \backslash\{k\}$. Since $\left(\cos (\varphi / 2) \sqrt{\sum \Psi_{i j}^{2}}-\Psi_{i j}\right)>0$ we write,

$$
\tilde{\psi}_{i j}>\frac{\Omega_{k}}{N_{p}-1} \frac{1}{\left(\cos (\varphi / 2) \sqrt{\sum \Psi_{i j}^{2}}-\Psi_{i j}\right) \Psi_{i j}}=f_{i j}
$$

Since $\tilde{\psi}_{i j}>0$ this condition will be always met when $f_{i j} \leq 0$. However a stronger condition needs to be found as $f_{i j}>0$.
Since $\log$ is monotonically increasing, and by substituting $\tilde{\psi}_{i j}$ expression,

$$
\begin{array}{r}
\sqrt{1-\rho^{2}} R_{i j}-(1-\rho)\left[G_{i j}\right]_{\mathrm{dB}}>\left[f_{i j}\right]_{\mathrm{dB}} \\
R_{i j}>\frac{1}{\sqrt{1-\rho^{2}}}\left((1-\rho)\left[G_{i j}\right]_{\mathrm{dB}}+\left[f_{i j}\right]_{\mathrm{dB}}\right)
\end{array}
$$

Therefore, when (42) is satisfied the off-grid error cannot be corrected. The probability of failure to recover is,

$$
Q\left(\frac{(1-\rho)\left[G_{i j}\right]_{\mathrm{dB}}+\left[f_{i j}\right]_{\mathrm{dB}}}{\sigma_{g}^{2} \sqrt{1-\rho^{2}}}\right)
$$

Also it should be noted that algebraically there is the possibility that $\Omega_{k}<0$ for all $k$. However in the problem of interest, unless all reference points are closely packed in space, some $\Psi_{i j}$ values are substantially larger than others. Therefore the probability of $\Omega_{k}<0$ for all $k$ is zero. Hence,

$$
1-\prod_{i=1}^{N_{p} \backslash\{k\}} Q\left(\frac{(1-\rho)\left[G_{i j}\right]_{\mathrm{dB}}+\left[f_{i j}\right]_{\mathrm{dB}}}{\sigma_{g}^{2} \sqrt{1-\rho^{2}}}\right)
$$

is the upper bound for the probability of successful recovery.

\section{B. Illustration of the basis vector rotation concept}

The fundamental principle behind the reconstruction algorithm is illustrated in Fig. 3 for a 3-dimensional scenario. In Fig. 3a we show a vector that has been projected onto two basis vectors. The residue in the third dimension is shown as a vector in red colour. This residue could be due to basis mismatch or due to the presence of an actual vector component in that dimension.

Consider the basis vector 1 and residue. Consider the intersection between the cone within which perturbed basis vector 1 sits and the plane that contains the residue and basis vector 1 . Any perturbation that produces a vector component in this plane contributes to the residue. Note that in the worst case scenario the basis vector could have been on the axis of intersection between the lateral cone surface and the plane that contains the residue and basis vector 1 .

Therefore, by allowing the basis vector 1 to move in the direction of the residue, we increase the length of vector projection onto the rotated basis vector while decreasing the length of the residue. This is shown in Fig. 3b. Note that the length of blue vector (basis vector 1) has grown while the length of red vector (residue) has shrunk.

This step has been repeated for the basis vector 2 and the residue in Fig. 3c. Therefore, the result is the smallest possible residue vector after providing for basis mismatch.

\section{Reconstruction algorithm}

When the condition in Theorem 2 is met, it is possible to reconstruct the $P_{t}^{*}$ vector that contains the locations and transmit powers of $k$ incumbents. We build on the well known OMP algorithm and the improvement to OMP proposed in [22].

Consider the scenario of attempting to solve (13) for $P_{t}^{*}$ using OMP. In each iteration of the algorithm, it discovers 


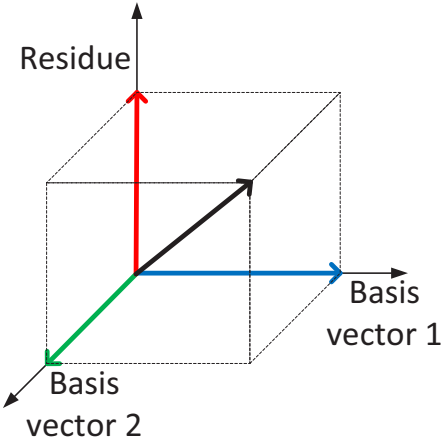

(a)

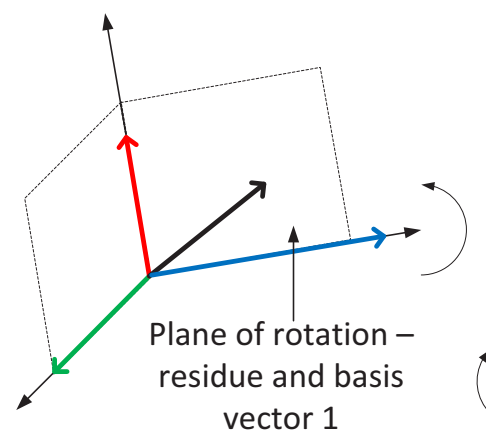

(b)

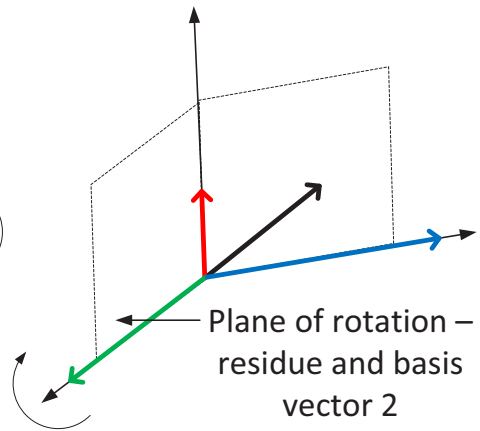

(c)

Fig. 3: Perturbation reduction by controlled rotation of basis vectors. (a) vector decomposition (b) rotation of basis vector 1 and residue (c) rotation of basis vector 2 and residue

one non-zero element in $P_{t}^{*}$. If the number of incumbents present is $k$, the vector $P_{t}^{*}$ should have $k$ non-zero elements (hence the algorithm will run for $k$ iterations). Consider the $n$th $(\leq k)$ iteration of OMP. At this point the algorithm has decided there are $n-1$ non-zero elements in $P_{t}^{*}$ i.e. pick $n-1$ columns of $\Phi \Psi$ as the signal support. The residue vector is calculated in the begining of $n$th iteration as follows,

$$
\gamma_{n}=P_{s}-\sum_{j \in \lambda_{n-1}}[\Phi \Psi]_{j}\left[P_{t}^{*}\right]_{j}
$$

where $\lambda_{n-1}$ is a set of column numbers of $\Phi \Psi$ that corresponds to non-zero elements in $P_{t}^{*}$ in $(n-1)$ th iteration. If the residue is below a predetermined threshold (i.e. $\gamma_{n} \leq \varepsilon$ ) the algorithm will not proceed to another iteration. In the ideal scenario where the measurement process uses $\Phi \Psi$ as shown in (6), this residue is a result of not having selected all $k$ basis vectors at $n$th $(\leq k)$ iteration and additive noise. Although OMP has been proved to be effective in the ideal scenario [21], the measurement process using $\Phi \tilde{\Psi}$ affects the performance significantly.

In the non-ideal scenario where the MCDs are off-grid and measurement process is given by (12), the residue in each iteration is also a result of basis mismatch in addition to the causes mentioned previously. Our objective is to reduce the impact of basis mismatch by allowing $[\Phi \Psi]_{j}$ (where $j \in \lambda_{n-1}$ ) vectors to rotate in the direction of $\gamma_{n}$ within predetermined boundaries. By making this provision we reduce the portion of the residue resulted by off-grid nodes being carried forward to the next iteration. Let the adjusted set of basis vectors be $\left\{[\psi]_{j} \mid j \in \lambda_{n-1}\right\}$. The residual when the adjusted basis is used can be written as,

$$
\tilde{\gamma}_{n}=P_{s}-\sum_{j \in \lambda_{n-1}}[\psi]_{j}\left[P_{t}^{*}\right]_{j}
$$

In [22, Th. 1], the authors show that the residual error $\tilde{\gamma}_{n}$ is bounded as,

$$
\left\|\tilde{\gamma}_{n}\right\|_{2} \leq\left\|\gamma_{n}\right\|_{2}\left(1-\frac{1}{\left\|\gamma_{n}\right\|_{2}} \sum_{j=1}^{n-1}\left|\left[P_{t}^{*}\right]_{j}\right| \tan \left(\vartheta_{j}\right)\right)
$$

when vector $j$ is rotated by $\vartheta_{j}$. We use this bound to develop a reconstruction algorithm that provides for the error caused by off-grid MCDs.

Theorem 4: The magnitude of the residual $\left\|\tilde{\gamma}_{n}\right\|_{2}$ is upper bounded by $\varepsilon$ when each basis vector $[\Phi \Psi]_{j}$ is rotated in the direction of $\gamma$, by an angle

$$
\vartheta_{j}=\arctan \left(\frac{\left\|\gamma_{n}\right\|_{2}-\varepsilon}{(n-1)\left|\left[P_{t}^{*}\right]_{j}\right|}\right)
$$

Proof: By substituting (48) into (47) we obtain,

$$
\begin{aligned}
\left\|\tilde{\gamma}_{n}\right\|_{2} & \leq\left\|\gamma_{n}\right\|_{2}\left(1-\frac{1}{\left\|\gamma_{n}\right\|_{2}} \sum_{j=1}^{n-1}\left|\left[P_{t}^{*}\right]_{j}\right| \frac{\left\|\gamma_{n}\right\|_{2}-\varepsilon}{(n-1)\left|\left[P_{t}^{*}\right]_{j}\right|}\right) \\
& \leq\left\|\gamma_{n}\right\|_{2}\left(1-\frac{1}{\left\|\gamma_{n}\right\|_{2}}\left(\left\|\gamma_{n}\right\|_{2}-\varepsilon\right)\right) \\
& \leq \varepsilon
\end{aligned}
$$

Therefore, by allowing each basis vector $[\Phi \Psi]_{j}$ to rotate by an angle of $\vartheta_{j}$ in the direction of $\gamma_{n}$ as proposed in Theorem 4 guarantees that residual $\left\|\tilde{\gamma}_{n}\right\|_{2} \leq \varepsilon$. Simple geometry proves that rotated vector $\psi_{j}$ can be found as [22, Eq. (8)],

$$
[\psi]_{j}=[\Phi \Psi]_{j} \cos \left(\vartheta_{j}\right)+\frac{\gamma}{\|\gamma\|_{2}} \operatorname{sgn}\left(P_{t}^{*}(j)\right) \sin \left(\vartheta_{j}\right)
$$

where sgn is the Sign function. Hence Algorithm 1 is proposed to solve (13) while minimising the impact of off-grid MCDs.

Algorithm 1 starts by initialising the residual vector $\gamma$ and incumbent locations/transmit power vector $P_{t}^{*}$. In steps 3-6 traditional OMP steps are performed. The actual channel to the MCDs $\Phi \tilde{\Psi}$ is not known to the algorithm, but the channels to the nearest reference points $\Phi \Psi$ are known. In the $i$ th iteration Line 2 checks if the residue $\|\gamma\|_{2}>\varepsilon$, if so it runs the algorithm for another iteration. As mentioned previously, $\|\gamma\|_{2}>\varepsilon$ could be due to $i<k$ or $\Phi \tilde{\Psi}$ being unknown to the algorithm. Steps 8-10 prevent the algorithm proceeding into another iteration if the residue is due to a basis mismatch. This is accomplished by allowing each basis vector to move in the 
Algorithm 1 Proposed incumbent location and transmit power detection algorithm

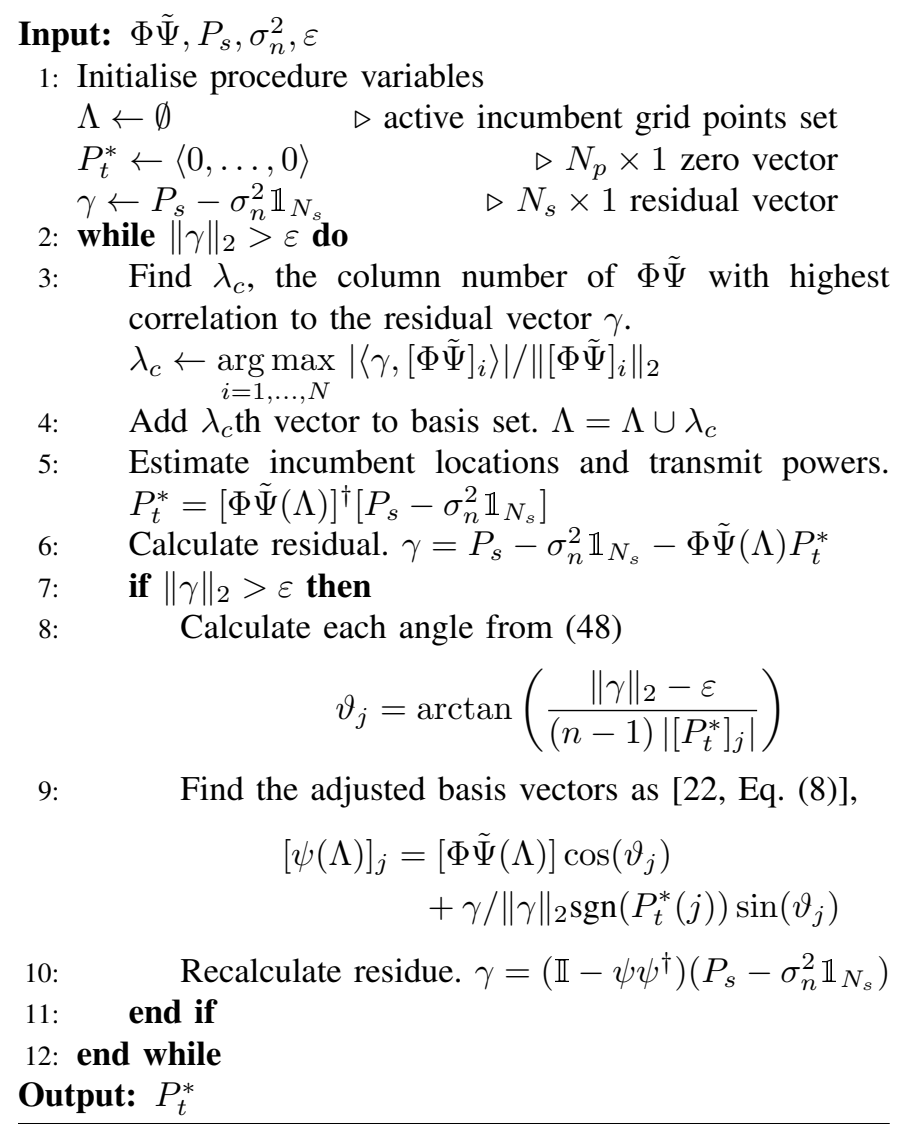

direction of $\gamma$ by an angle of $\vartheta_{j}$. A rotated basis vector $[\psi]_{j}$ would in fact be a linear combination of vectors $[\Phi \Psi]_{j}$ and $\gamma$. Since $[\psi]$ the set of basis vectors have absorbed a portion of $\gamma$ into themselves, the resulting residue is of a smaller magnitude (i.e. $\gamma_{i+1}<\gamma_{i}$ ). Therefore, the convergence of the algorithm is assured.

\section{Numerical Evaluation}

In this section we evaluate the performance of the proposed algorithm in the context of a LSA network operating in 2300$2400 \mathrm{MHz}$ band (3GPP band 40 [27]). We consider a scenario in which LSA licensee is a mobile network operator and the incumbent is a network of Electronic News Gathering (ENG) devices (a detailed list of LSA use cases can be found in [15]). ENG refers to the rapid deployment of wireless links to cover breaking news in an unplanned manner for a short period of time [28]. Especially we consider a ENG application in which mobile wireless video cameras with omnidirectional antennas broadcast a video link to a Cam-CCU as illustrated in [28, Fig. 2].

Our proposed approach can be used on any layout of reference points. However due to simplicity in geometry and more suitability for an urban environment, we consider a $15 \times 15$ square grid. The separation between grid points is $10 \mathrm{~m}$, covering $150 \times 150 \mathrm{~m}$ area. This area covers the size of a sports stadium, hence it aligns with the use case in [28,
Fig. 2, Table 15]. On this square grid layout, the $j$ th reference point can be mapped to (row, column) coordinates as: $j \rightarrow$ $\left(\left\lceil j / \sqrt{N_{p}}\right\rceil, j-\sqrt{N_{p}}\left(\left\lceil j / \sqrt{N_{p}}\right\rceil-1\right)\right.$, where $N_{p}=15 \times 15$.

In our system model, the LSA repository flags a large geographical region where potentially active incumbents are present. A mobile network operator with a LSA license to access incumbent spectrum in this region uses MCDs (UEs and/or dedicated measurement devices) to collect measurements of the incumbent signals. Particularly, the MCDs inform the Received Signal Strength (RSS) of the incumbent signal (combined signal from all incumbent transmitters) and the geolocation where the measurement was taken. A central entity in the mobile network processes these measurements to find the geolocation and power level of the incumbent transmitters.

In practice the incumbent RSS can be measured using the quiet period concept. In a TD-LTE system this can be achieved by blanking out a downlink subframe in each TDD frame. LSA licensee MCDs can collect the RSS of the incumbent signal during the blanked subframe. Mean of the RSS can be calculated over multiple radio frames and sent to the base station.

In this analysis we consider an urban environment. To simulate the incumbent signal propagation we utilise the WINNER II model used in International Telecommunications Union (ITU) model [25]. The hexagonal layout Non-line of sight (NLos) Urban Microcells (UMi) pathloss model was used to calculate $L_{i j}$ as [25],

$$
1 / L_{i j}=36.7 \log _{10}\left(d_{i j}\right)+22.7+26 \log _{10}\left(f_{c}\right)
$$

where $f_{c}$ is the centre frequency. The shadow fading follows a log-normal distribution i.e. $G_{i j} \sim \mathcal{N}\left(0, \sigma_{g}^{2}=4\right)$. Additionally, an exponentially decaying correlation is assumed in space. The ITU model used to capture correlation is (15) [25, Eq. (6)], when line of sight is not available the decorrelation distance $d_{c}=13 \mathrm{~m}$ [25]. The transmission parameters of the incumbent follow [28, Table 15].

Simulations were run for 10,000 iterations. We compare our proposed approach against the OMP algorithm [21], POMP algorithm [22] and convex optimisation solution obtained through Matlab. All algorithms were run until the residue became small i.e. $\|\gamma\|_{2} \leq \varepsilon=10^{-9}$.

\section{A. Detected number of incumbents}

In Fig. 4 we show the number of incumbents detected by OMP, POMP and the proposed algorithm. We vary the number of incumbents $k$ in the system from 2 to 12 , having fixed all other parameters. This experiment was repeated for different off grid errors $\Delta d$. If the algorithm detects all incumbents accurately, we expect to have detected $k$ incumbents each time. Ideally a straight line with a gradient of 1 is expected in Fig.4.

When the actual number of incumbents is in the order of 24, both POMP and the proposed algorithm return the accurate number of incumbents for all $\Delta d$. OMP is close to the ideally expected result when $\Delta d=0.5 \mathrm{~m}$ and $k=2$. However in practice, $\Delta d$ is expected to be in the order of few meters. OMP detects over twice as the actual number of incumbents present. This is due to the mismatch of the basis used in the $\ell_{1}$ 


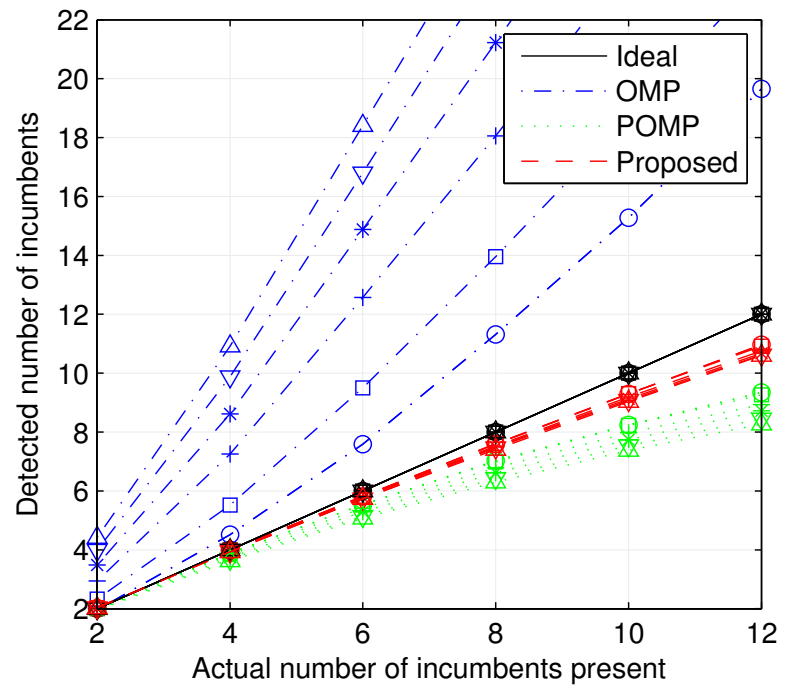

Fig. 4: Detected and the actual number of incumbents for different off-grid errors $\Delta d=0.5,1,2,3,4,5 \mathrm{~m}$. The markers are $(\bigcirc: \Delta d=0.5),(\square: \Delta d=1),(+: \Delta d=2),(*: \Delta d=$ $3),(\nabla: \Delta d=4),(\Delta: \Delta d=5)$.

norm minimisation process. Having too many non-zero entries in $P_{t}$ results in a higher False Alarm.

When $\Delta d$ changes, OMP produces substantially different results. The proposed approach is more robust against offgrid errors. However, when the number of incumbents was increased the performance of all three algorithms degrade. As discussed in [29], the minimum number of measurements required for exact recovery of $P_{t}$ is a linear function of the number of incumbents i.e. $N_{s}>f(k)$. Therefore when the number of incumbents increases, the required number of sensors also increases. The number of sensors taking measurements in this simulation was fixed, which explains the degradation of performance. However, the proposed algorithm clearly outperforms others under consideration.

\section{B. False Alarm and Missed Detection vs number of MCDs}

The Missed Detection (MD) and False Alarm (FA) probabilities are shown in Fig. 5 and Fig. 6 respectively. The incumbents could be at any reference point on the $15 \times 15$ grid. We estimate the RSS of the incumbent signal based on $P_{t}^{*}$ and compare against a threshold $P_{t h}$ to determine if the $i$ th point is encountering MD or FA as follows,

$$
\begin{aligned}
\operatorname{MD}(i) & =\sum_{j=1}^{N_{p}} \Psi_{i j} P_{t}^{*}(j)<P_{t h} \mid \sum_{j=1}^{N_{p}} \Psi_{i j} P_{t}(j)>P_{t h} \\
\operatorname{FA}(i) & =\sum_{j=1}^{N_{p}} \Psi_{i j} P_{t}^{*}(j)>P_{t h} \mid \sum_{j=1}^{N_{p}} \Psi_{i j} P_{t}(j)<P_{t h}
\end{aligned}
$$

The IEEE 802.22 standard states the decision threshold should fall within the range $-120 \leq P_{t h} \leq-10 \mathrm{dBm}$ [30]. The threshold is said to be subjective, but it should fall within the above range. Therefore, in this simulation we choose $P_{t h}=-70 \mathrm{dBm}$. We simulate the challenging situation of

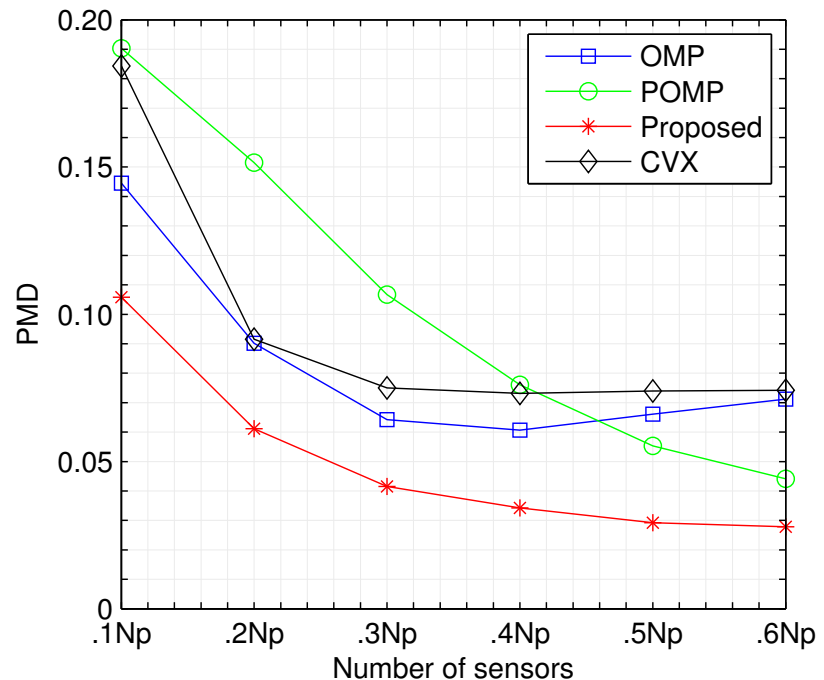

Fig. 5: Probability of Missed Detection vs the number of measurements. We compare the proposed algorithm, OMP [21], POMP [22] and the $\ell_{1}$-minimisation solution (CVX)

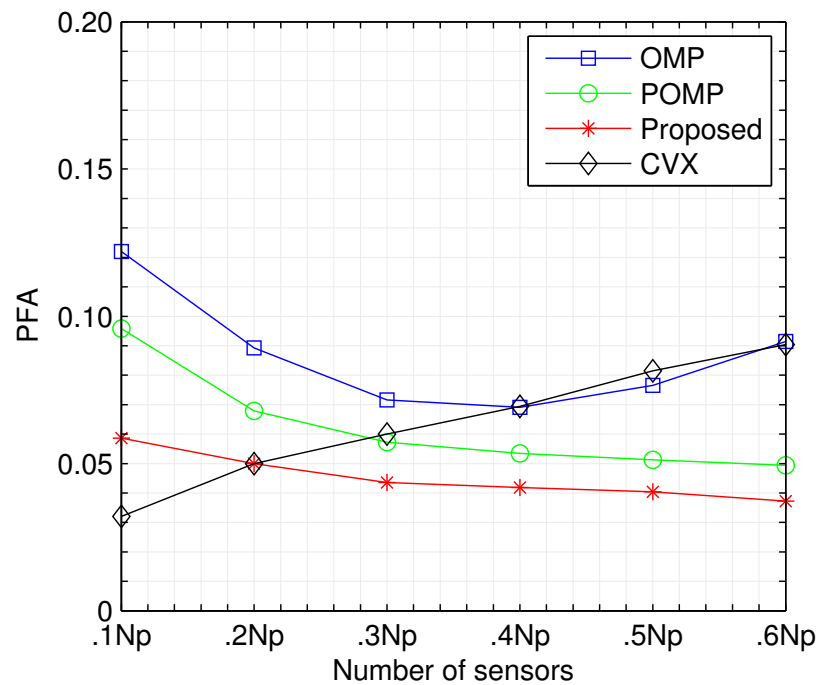

Fig. 6: Probability of False Alarm vs the number of measurements.

having 16 incumbents (the largest number of incumbents in the simulations) sparsely distributed in space.

To accurately determine the mean MD and FA probabilities we pad the grid. An incumbent located on the border of $15 \times 15$ grid clearly affects an area outside the grid. Therefore calculating the MD and FA probabilities only in the area covered by $15 \times 15$ grid is not sufficient. Therefore the grid layout was padded to extend the area to a $21 \times 21$ grid. From (54) and (55) MD and FA were determined for each reference point. Probability was calculated as the number of MD/FA occurrences divided by the total number of points. Mean of the probability was calculated over all iterations.

The Fig. 5 and Fig. 6 show the mean probability of MD (PMD) and mean probability of FA (PFA), for different MCD densities. Clearly the proposed approach performs better 
TABLE I: Feasible regions of operation for both PMD and PFA $<0.05$. $k$ : number of incumbents and $N_{s}$ : number of measurements. This table summarises the results in Fig. 7 and 8.

\begin{tabular}{|l|c|c|}
\hline Approach & $\begin{array}{c}\text { Number of in- } \\
\text { cumbents }(\mathbf{k})\end{array}$ & $\begin{array}{c}\text { Number of } \\
\text { MCDs }\left(\mathbf{N}_{\mathbf{s}}\right)\end{array}$ \\
\hline CVX & $<4$ & $.25 N_{p}-.35 N_{p}$ \\
\hline OMP & $<3$ & $.35 N_{p}-.60 N_{p}$ \\
\hline POMP & $<3$ & $>.35 N_{p}$ \\
\hline Proposed & $<3$ & $.09 N_{p}-.15 N_{p}$ \\
& $4-12$ & $.15 N_{p}-.35 N_{p}$ \\
& Tested up to 16 & $>.35 N_{p}$ \\
\hline
\end{tabular}

than others under consideration when the number of MCDs $N_{s} \geq .2 N_{p}$. In practice MCD density is expected to fall within the range $.3 N_{p}$ to $.4 N_{p}$. In this range the proposed approach improves the PMD by at least $40 \%$ and the PFA by at least $30 \%$.

Further, it should be noted when the number of sensors $N_{s}<.2 N_{p}$ the PFA in the CVX approach is lower than all other approaches. Also we notice that the PMD of CVX approach is substantially high within this region. Therefore Fig. 5 and Fig. 6 suggest that CVX terminates prematurely without discovering all incumbents when $N_{s}<.2 N_{p}$, which results in low FA and high MD.

\section{False Alarm and Missed Detection vs number of incum- bents and MCDs}

Fig. 7 and Fig. 8 show the impact of the number of incumbents and measurements. A darker colour implies low PMD/PFA, hence preferable. Clearly the proposed algorithm produces a lower PMD/PFA over a wide range of $k$ and $N_{s}$. To quantify the results, consider the region in Fig. 7, 8 where both PMD and PFA $<0.05$. Table I shows how CVX, OMP and POMP have a limited feasible region in comparison to the proposed approach. Generally, the accuracy of $\ell_{1}$ minimisation techniques increases with $N_{s}$. However the accuracy decreases in the high $N_{s}$ region since both CVX and OMP make no provision for off-grid error. This is illustrated in Table I.

Consider the region where both PMD and PFA $<0.05$ and the number of MCDs taking measurements is at least $.35 N_{p}$ which is a more practical region of operation. Fig. 7 and 8 suggest that the proposed algorithm can accommodate up to 16 incumbents. In contrast, under identical conditions POMP, CVX and OMP can accommodate only up to 4 incumbents. Therefore the number of incumbents that can be accommodated has been improved by 4 times under typical operating conditions of the LSA network.

\section{Distance between signal supports}

To compare the overall performance we use the distance between the expected and estimated signal supports. The distance is determined as,

$$
1-\frac{\left|\left\{P_{t}^{*} \mapsto \Phi \tilde{\Psi}\right\} \cap\left\{P_{t} \mapsto \Phi \Psi\right\}\right|}{\max \left\{\left|P_{t}^{*} \mapsto \Phi \tilde{\Psi}\right|,\left|P_{t} \mapsto \Phi \Psi\right|\right\}}
$$

where $\left\{P_{t}^{*} \mapsto \Phi \tilde{\Psi}\right\}$ is the set of column vectors in $\Phi \tilde{\Psi}$ that map to non-zero elements in $P_{t}^{*}$. The $|\cdot|$ operator refers to the cardinality of a set. Therefore, the distance in (56) measures the number of column vectors of $\Phi \Psi$ incorrectly included or incorrectly excluded in the chosen support as a ratio to the total number of column vectors. A large distance between signal supports intuitively lead to limited accuracy of the $\ell_{1}$ minimisation process.

As shown in Fig. 9 both CVX and OMP result in highly distant supports as they make no provision for off-grid nodes. The proposed approach can accommodate on average 50\% more incumbents or $33 \%$ less measurements in comparison to POMP.

\section{CONCLUSION}

Based on our previous work [11], [12], we presented a discrete space $\ell_{1}$-norm minimisation solution for detecting the location and transmit power of multiple incumbents. We exploited the sparsity of incumbents in spatial domain through geolocation tagged energy detection measurements. When using a discrete space model in practice, the impact of offgrid measurement devices should be considered. This effect can be contemplated as a limitation of the accuracy in selfgeolocalisation. We capture the off-grid error as a basis mismatch in the $\ell_{1}$-norm minimisation problem. We analytically proved the channel matrix meets the RIP criterion under this system model, when the number of measurement nodes is sufficiently large. We found the necessary condition and the upper bound for the probability of eliminating the impact of limited geolocation tagging accuracy, that must be satisfied by any generic algorithm to recover from the basis mismatch error. When this criterion was met, we proposed an extension to the well known OMP algorithm. In any $i$ th iteration the residue of OMP is due to $i$ being less than the number of incumbents and basis mismatch. We reduce the latter by allowing a selected set of basis vectors to rotate by a controlled angle. This algorithm was evaluated in a LSA cellular network operating in $2300-2400 \mathrm{MHz}$ band. Numerical results showed a substantial improvement in incumbent detection, false alarm probability and missed detection probability.

\section{APPENDIX A \\ PROOF OF THEOREM 1}

The shadowing process typically follows a log-normal distribution i.e. $G_{i j} \sim \exp \left(\mathcal{N}\left(0, \sigma_{g}^{2}\right)\right)$. Hence the non-zero elements,

$$
\begin{aligned}
\frac{\eta G_{i j} L_{i j} v_{j}}{\|v\|} & =G_{i j} \exp \left(\log \frac{\eta L_{i j} v_{j}}{\|v\|}\right) \\
& \sim \exp \left(\mathcal{N}\left(\log \frac{\eta L_{i j} v_{j}}{\|v\|}, \sigma_{g}^{2}\right)\right)
\end{aligned}
$$

where $\eta$ is the normalisation constant defined later in this section. The reference points are far apart in space, therefore $G_{i j}$ can be considered independent for each reference point. Hence each element in $\Phi \Psi v$ vector is a sum of independent $\log$ normal distributions. The resulting distribution can be approximated by another log-normal distribution using the 


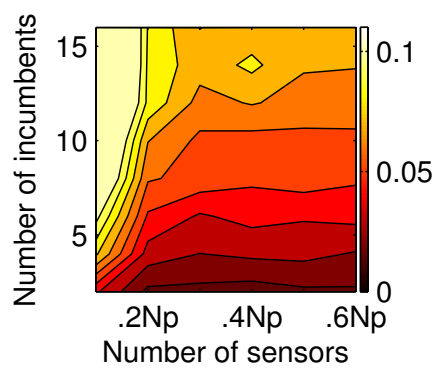

(a)

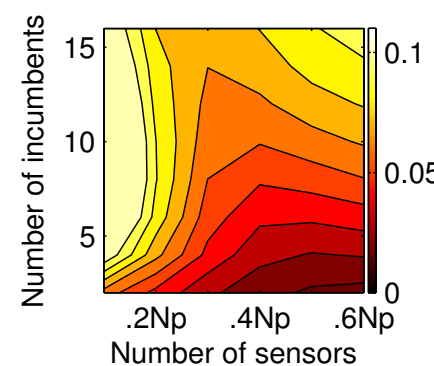

(b)

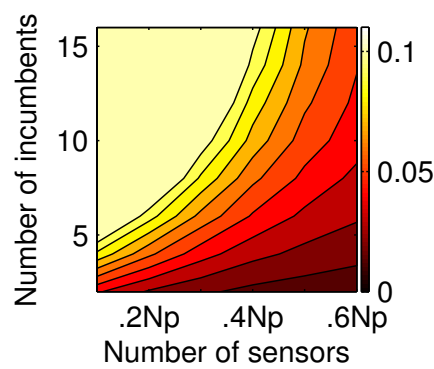

(c)

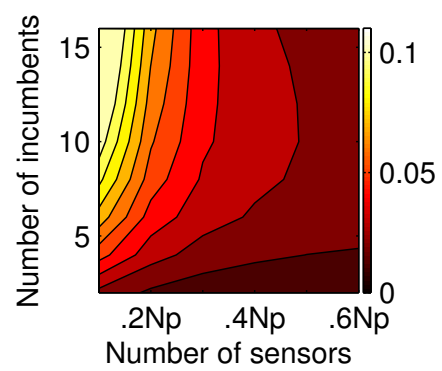

(d)

Fig. 7: Probability of missed detection vs number of incumbents and number of sensors. Probability has been calculated as $N_{m d} / N_{p}$ where $N_{m d}$ is the number of discrete grid points where incumbent was left undetected and $N_{p}$ is the total number of grid points (a) $\ell_{1}$-norm convex optimisation (b) OMP (c) POMP (d) proposed approach

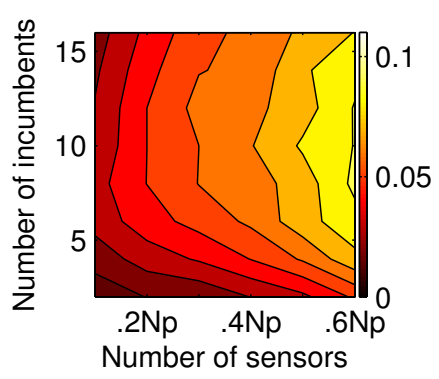

(a)

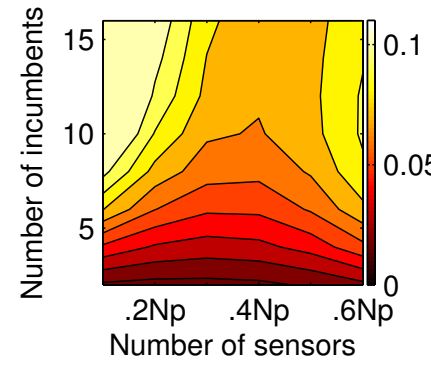

(b)

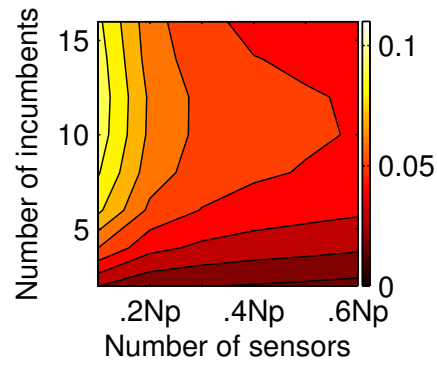

(c)

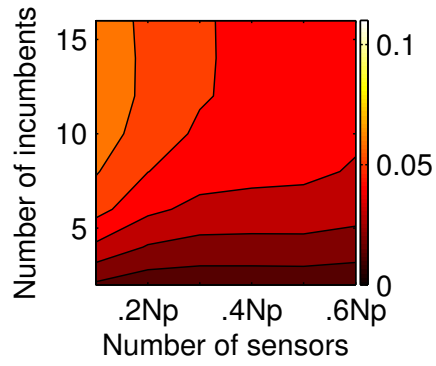

(d)

Fig. 8: Probability of false alarm vs number of incumbents and number of sensors. Probability has been calculated as $N_{f a} / N_{p}$ where $N_{f a}$ is the number of discrete grid points where incumbent was left undetected and $N_{p}$ is the total number of grid points (a) $\ell_{1}$-norm convex optimisation (b) OMP (c) POMP (d) proposed approach

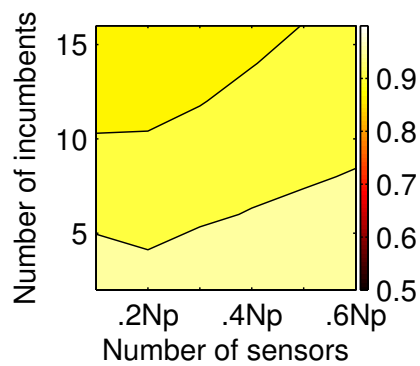

(a)

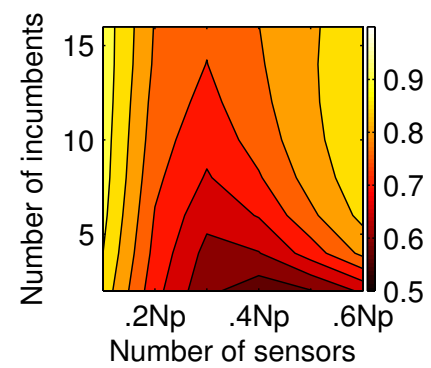

(b)

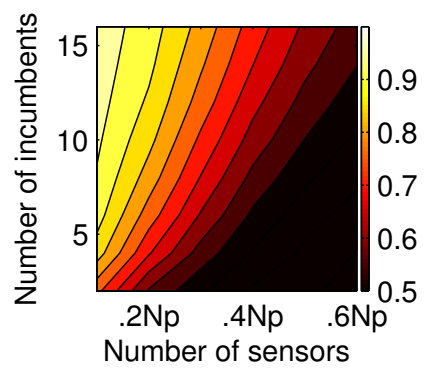

(c)

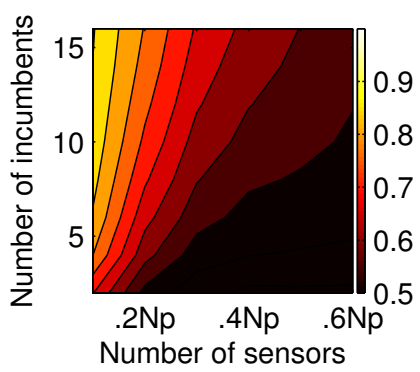

(d)

Fig. 9: Distance between signal supports (defined in (56)) vs number of incumbents and number of sensors. Distance between supports is calculated as $1-\left|\left\{P_{t}^{*} \mapsto \Phi \tilde{\Psi}\right\} \cap\left\{P_{t} \mapsto \Phi \Psi\right\}\right| / \max \left\{\left|P_{t}^{*} \mapsto \Phi \tilde{\Psi}\right|,\left|P_{t} \mapsto \Phi \Psi\right|\right\}$, where $P_{t}^{*} \mapsto \Phi \tilde{\Psi}$ are the column vectors of $\Phi \tilde{\Psi}$ that are being mapped to non-zero elements of $P_{t}^{*}$ and $|\cdot|$ is the cardinality of a set. (a) $\ell_{1}$-norm convex optimisation (b) OMP (c) POMP (d) proposed approach

Fenton-Wilkinson method [31], [32, Ch. 3]. As $v$ is essentially picking some of the random values in $\Phi \Psi$, the mean and variance of $i$ th element in $\Phi \Psi v$ vector are,

$$
\begin{aligned}
\mu_{i} & =\frac{1}{2}\left(\sigma_{g}^{2}-\sigma_{i}^{2}\right)+\log \left(\sum_{j=1}^{N_{p}} \frac{\eta L_{i j} v_{j}}{\|v\|}\right) \\
\sigma_{i}^{2} & =\log \left(\left(\exp \sigma_{g}^{2}-1\right) c_{i}+1\right)
\end{aligned}
$$

where,

$$
\begin{aligned}
c_{i}=\frac{\sum_{j=1}^{N_{p}}\left(\frac{\eta L_{i j} v_{j}}{\|v\|}\right)^{2}}{\left(\sum_{j=1}^{N_{p}} \frac{\eta L_{i j} v_{j}}{\|v\|}\right)^{2}} \\
=\frac{\sum_{j=1}^{N_{p}}\left(L_{i j} v_{j}\right)^{2}}{\left(\sum_{j=1}^{N_{p}} L_{i j} v_{j}\right)^{2}}
\end{aligned}
$$


Since $\sigma_{g}^{2} \gg 1$ is a large number, we make the following approximation.

$$
\sigma_{i}^{2} \approx \log \left(1+c_{i} \exp \sigma_{g}^{2}\right)
$$

Therefore $[\Phi \Psi v]_{i}^{2}$ terms are log-normally distributed as follows,

$$
\begin{aligned}
& \mu_{i}=\left(\sigma_{g}^{2}-\sigma_{i}^{2}\right)+2 \log \left(\sum_{j=1}^{N_{p}} \frac{\eta L_{i j} v_{j}}{\|v\|}\right) \\
& \sigma_{i}^{2}=4 \log \left(1+c_{i} \exp \sigma_{g}^{2}\right)
\end{aligned}
$$

Similarly as before we find an approximate $\|\Phi \Psi v\|_{2}^{2}$ with a log-normal distribution having the following parameters,

$$
\begin{aligned}
\mu & =\frac{1}{2}\left(\sigma_{i}^{2}-\sigma^{2}\right)+\log \left(\sum_{i=1}^{N_{s}} \mu_{i}\right) \\
\sigma^{2} & =\log \left(1+c\left(1+c_{i} \exp \sigma_{g}^{2}\right)^{4}\right)
\end{aligned}
$$

where

$$
c=\frac{\sum_{i=1}^{N_{s}} \exp \left(2 \mu_{i}\right)}{\left(\sum_{i=1}^{N_{s}} \exp \left(\mu_{i}\right)\right)^{2}}
$$

Pick $\eta=\eta^{*}$ such that $\mu=1$. We consider the specific form of the Chernoff-Hoeffding bound used in [33, Eq. (23)]. When $\mu=1$, we write the following inequality

$$
\begin{array}{r}
\operatorname{Pr}\left\{\left|\frac{\|\eta \Phi \Psi v\|_{2}^{2}}{\|v\|_{2}^{2}}-1\right|>\delta\right\} \leq 2 \exp \left(\frac{-\delta^{2}}{4 \sigma^{2}}\right) \\
\Rightarrow \operatorname{Pr}\left\{\left|\frac{\|\eta \Phi \Psi v\|_{2}^{2}}{\|v\|_{2}^{2}}-1\right|>\delta\right\} \\
\leq 2 \exp \left(\frac{-\delta^{2}}{4 \log \left(1+c\left(1+c_{i} \exp \sigma_{g}^{2}\right)^{4}\right)}\right)
\end{array}
$$

Since $\log x \leq x-1$,

$$
\leq 2 \exp \left(\frac{-\delta^{2}}{4 c\left(1+c_{i} \exp \sigma_{g}^{2}\right)^{4}}\right)
$$

Consider the scenario of having a large set of reference points $\left(N_{p} \gg N_{s}\right)$. As the pathloss coefficient exponentially decreases with distance (i.e. $L_{i j} \propto 1 / d^{\alpha}$ ), a large subset of elements in any column vector of $\Phi \Psi$ take similar small values. Although the variance of $\eta^{*} L_{i j} v_{j} /\|v\|$ terms are dependent on $v$, the means $\mu_{i}$ vary less with $i$. Therefore we write,

$$
\begin{aligned}
& 0<\frac{1}{N_{s}} \sum_{j=1}^{N_{s}}\left(\exp \left(\mu_{i}\right)\right)^{2}-\left(\frac{1}{N_{s}} \sum_{j=1}^{N_{s}} \exp \left(\mu_{i}\right)\right)^{2}<\epsilon \\
& \Rightarrow 0<c-\frac{1}{N_{s}}<\dot{\epsilon}
\end{aligned}
$$

for some small $\epsilon, \dot{\epsilon} \in(0,1)$. Since the number of $k$ dimensional subspaces of $\Phi \Psi$ is $\left(\begin{array}{c}N_{p} \\ k\end{array}\right)$,

$$
\begin{aligned}
\operatorname{Pr}\left\{\left|\frac{\|\eta \Phi \Psi v\|_{2}^{2}}{\|v\|_{2}^{2}}-1\right|>\delta\right\}\left(\begin{array}{c}
N_{p} \\
k
\end{array}\right) \\
\leq 2 \exp \left(\frac{-\delta^{2} N_{s}}{4\left(1+c_{i} \exp \sigma_{g}^{2}\right)^{4}}\right)\left(\frac{N_{p} \exp 1}{k}\right)^{k} \\
\leq 2 \exp \left(\frac{-\delta^{2} N_{s}}{4\left(1+c_{i} \exp \sigma_{g}^{2}\right)^{4}}+k \log \frac{N_{p}}{k}+k\right)
\end{aligned}
$$

Inequality in (75) implies the value of the random variable is strongly concentrated about its expected values [34]. For a sufficiently large $N_{s}\left(>k \log \left(N_{p} / k\right)\right)$,

$$
\operatorname{Pr}\left\{\left|\frac{\|\eta \Phi \Psi v\|_{2}^{2}}{\|v\|_{2}^{2}}-1\right|>\delta\right\}\left(\begin{array}{c}
N_{p} \\
k
\end{array}\right) \rightarrow 0
$$

Therefore, we prove that there exist constants $a_{1}, a_{2}>0$ such that the matrix $\Phi \Psi$ holds RIP condition for any $N_{s} \geq$ $a_{1} k \log \left(N_{p} / k\right)$ with probability $\geq 1-\exp \left(-a_{2} N_{s}\right)$ which tends to 1 [34, Th. 5.2].

\section{REFERENCES}

[1] W. Yin, P. Ren, Q. Du, and Y. Wang, "Delay and throughput oriented continuous spectrum sensing schemes in cognitive radio networks," Wireless Communications, IEEE Transactions on, vol. 11, no. 6, pp. 2148-2159, Jun. 2012.

[2] W. Han, J. Li, Z. Tian, and Y. Zhang, "Dynamic sensing strategies for efficient spectrum utilization in cognitive radio networks," Wireless Communications, IEEE Transactions on, vol. 10, no. 11, pp. 3644-3655, Nov. 2011.

[3] Y.-C. Liang, Y. Zeng, E. Peh, and A. T. Hoang, "Sensing-throughput tradeoff for cognitive radio networks," Wireless Communications, IEEE Transactions on, vol. 7, no. 4, pp. 1326 -1337, Apr. 2008.

[4] W. Zhang, R. Mallik, and K. Letaief, "Optimization of cooperative spectrum sensing with energy detection in cognitive radio networks," Wireless Communications, IEEE Transactions on, vol. 8, no. 12, pp. $5761-5766$, Dec. 2009.

[5] ETSI Reconfigurable Radio Systems (RRS), "System requirements for operation of Mobile Broadband Systems in the $2300 \mathrm{MHz}-2400 \mathrm{MHz}$ band under Licensed Shared Access (LSA)," ETSI TS 103154 V1.1.1, Tech. Rep., Oct. 2014.

[6] Federal Communications Commission, "Amendment of the Commission Rules with Regard to Commercial Operations in the $3550-3650 \mathrm{MHz}$ Band," GN Docket No. 12-354, Tech. Rep., Apr. 2014.

[7] T. Xue, Y. Shi, and X. Dong, "A framework for location-aware strategies in cognitive radio systems," Wireless Communications Letters, IEEE, vol. 1, no. 1, pp. 30-33, Feb. 2012.

[8] A. Alaya-Feki, S. Ben Jemaa, B. Sayrac, P. Houze, and E. Moulines, "Informed spectrum usage in cognitive radio networks: Interference cartography," in Personal, Indoor and Mobile Radio Communications (PIMRC), 2008 IEEE 19th International Symposium on, pp. 1-5, Sep. 2008.

[9] J. van de Beek, T. Cai, S. Grimoud, B. Sayrac, P. Mahonen, J. Nasreddine, and J. Riihijarvi, "How a layered rem architecture brings cognition to today's mobile networks," Wireless Communications, IEEE, vol. 19, no. 4, pp. 17-24, Aug. 2012.

[10] G. Boccolini, G. Hernandez-Penaloza, and B. Beferull-Lozano, "Wireless sensor network for spectrum cartography based on kriging interpolation," in Personal Indoor and Mobile Radio Communications (PIMRC), 2012 IEEE 23rd International Symposium on, pp. 1565-1570, Sep. 2012.

[11] B. A. Jayawickrama, E. Dutkiewicz, I. Oppermann, G. Fang, and J. Ding, "Improved performance of spectrum cartography based on compressive sensing in cognitive radio networks," in International Conference on Communications (ICC), 2013 IEEE, pp. 5657-5661, Jun. 2013.

[12] B. A. Jayawickrama, E. Dutkiewicz, I. Oppermann, M. D. Mueck, and G. Fang, "Downlink power allocation algorithm for licence-exempt LTE systems using kriging and compressive sensing based spectrum cartography," in Global Communications Conference (GLOBECOM), 2013 IEEE, pp. 3766-3771, Dec. 2013. 
[13] B. A. Jayawickrama, E. Dutkiewicz, I. Oppermann, and M. D. Mueck, "Iteratively reweighted compressive sensing based algorithm for spectrum cartography in cognitive radio networks," in Wireless Communications and Networking Conference (WCNC), 2014 IEEE, pp. 719-724, Apr. 2014

[14] S. M. Yu and S.-L. Kim, "Optimal detection of spatial opportunity in wireless networks," Communications Letters, IEEE, vol. 15, no. 4, pp. 395-397, Apr. 2011.

[15] Electronic Communications Committee (ECC), "Results of the WG FM questionnaire to CEPT administrations on the current and future usage of frequency band 2300-2400 MHz," FM52(12)INFO2 FM(12)017rev1, Tech. Rep., Apr. 2012.

[16] H. Yilmaz, T. Tugcu, F. Alagöz, and S. Bayhan, "Radio environment map as enabler for practical cognitive radio networks," Communications Magazine, IEEE, vol. 51, no. 12, pp. 162-169, Dec. 2013.

[17] H. Yilmaz, C.-B. Chae, and T. Tugcu, "Sensor placement algorithm for radio environment map construction in cognitive radio networks," in Wireless Communications and Networking Conference (WCNC), 2014 IEEE, pp. 2096-2101, Apr. 2014.

[18] S. Gleichman and Y. Eldar, "Blind compressed sensing," Information Theory, IEEE Transactions on, vol. 57, no. 10, pp. 6958-6975, Oct. 2011.

[19] Z. Yang, C. Zhang, and L. Xie, "Robustly stable signal recovery in compressed sensing with structured matrix perturbation," Signal Processing, IEEE Transactions on, vol. 60, no. 9, pp. 4658-4671, Sep. 2012.

[20] J. Wormann, S. Hawe, and M. Kleinsteuber, "Analysis based blind compressive sensing," Signal Processing Letters, IEEE, vol. 20, no. 5 , pp. 491-494, May. 2013.

[21] J. Tropp and A. Gilbert, "Signal recovery from random measurements via orthogonal matching pursuit," Information Theory, IEEE Transactions on, vol. 53, no. 12, pp. 4655 -4666, Dec. 2007.

[22] O. Teke, A. Gurbuz, and O. Arikan, "Perturbed orthogonal matching pursuit," Signal Processing, IEEE Transactions on, vol. 61, no. 24, pp. 6220-6231, Dec. 2013.

[23] R. G. Baraniuk, "Compressive Sensing," Signal Processing Magazine, IEEE , vol.24, no.4, pp.118-121, Jul. 2007.

[24] C. Feng, S. Valaee, and Z. Tan, "Multiple target localization using compressive sensing," in Global Communications Conference (GLOBECOM), 2009 IEEE, pp. 1-6, Dec. 2009.

[25] Radiocommunication Sector of ITU, "Guidelines for evaluation of radio interface technologies for imt-advanced (report ITU-R M.2135-1)," International Telecommunication Union, Tech. Rep., Dec. 2009.

[26] M. Gudmundson, "Correlation model for shadow fading in mobile radio systems," Electronics Letters, vol. 27, no. 23, pp. $2145-2146$, Nov. 1991.

[27] 3GPP, "3rd Generation Partnership Project Technical Specification Group Radio Access Network, User Equipment (UE) radio transmission and reception (TDD),” 3GPP TS 25.102 V12.0.0, Tech. Rep., Sep. 2014.

[28] Radiocommunication Sector of ITU, "Tuning ranges and operational characteristics of terrestrial Electronic News Gathering (ENG), television outside broadcast (TVOB) and electronic field production (EFP) systems," ITU-R BT.2069-6, International Telecommunication Union, Tech. Rep., Feb. 2015.

[29] E. Candes and M. Wakin, "An introduction to compressive sampling," Signal Processing Magazine, IEEE, vol. 25, no. 2, pp. 21 -30, Mar. 2008.

[30] "IEEE Standard for Information technology- Local and metropolitan area networks- Specific requirements- Part 22: Cognitive Wireless RAN Medium Access Control (MAC) and Physical Layer (PHY) specifications: Policies and procedures for operation in the TV Bands," IEEE Std. 802.22-2011, pp. 1-680, 2011.

[31] N. Beaulieu, A. Abu-Dayya, and P. McLane, "Estimating the distribution of a sum of independent lognormal random variables," Communications, IEEE Transactions on, vol. 43, no. 12, pp. 2869-2873, Dec. 1995.

[32] G. Stüber, Principles of Mobile Communication. Springer, 2011.

[33] A. Vahid, M. A. Maddah-Ali, A. S. Avestimehr, "Capacity Results for Binary Fading Interference Channels With Delayed CSIT," Information Theory, IEEE Transactions on, vol.60, no.10, pp.6093-6130, Oct. 2014.

[34] R. Baraniuk, M. Davenport, R. DeVore, and M. Wakin, "A simple proof of the restricted isometry property for random matrices," Constructive Approximation, vol. 28, no. 3, pp. 253-263, Jan. 2008.

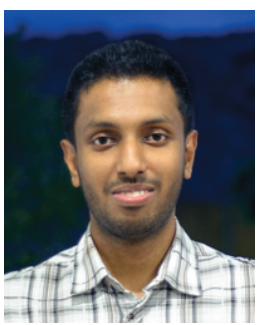

Beeshanga Abewardana Jayawickrama received the B.E. in Telecommunications Engineering (Hons. I) degree and Ph.D. in Electronic Engineering degree from Macquarie University, Sydney, Australia, in 2011 and 2015 respectively.

Following his Ph.D. he held a Research Associate position in the Department of Engineering at Macquarie University. He is currently a Lecturer in the School of Computing and Communications, University of Technology Sydney, Sydney, Australia. $\mathrm{He}$ has close industry relationships with Intel Labs and Commonwealth Scientific and Industrial Research Organisation (CSIRO). His research interests are resource allocation in wireless networks, cognitive radio, compressed sensing and cross-layer techniques.

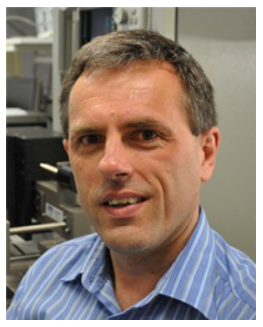

Eryk Dutkiewicz has over 25 years of industrial and academic research experience. He has conducted research and development with network operators and equipment manufacturing companies. Over the last ten years his research focused on quality of service mechanisms for wireless and mobile networks. From 1999 to 2004 he worked at Motorola where he managed a wireless research laboratory. Since returning to academia he has worked closely with industry partners including Intel, Motorola, Agere Systems Freescale, CSIRO, Zarlink and Infineon on projects involving ultra wideband technologies, wireless sensor networks, mesh networks, LTE and 5G networks. He currently works at the University of Technology Sydney where he is the Head of School of Computing and Communications. His research interests are in resource optimization for broadband communications networks and wireless medical body area networks. Prof. Dutkiewicz has also held visiting appointments at the Chinese Academy of Sciences in Beijing, Shanghai Jiao Tong University, City University of Hong Kong and Coventry University. He is an author of over 200 research papers and several book chapters.

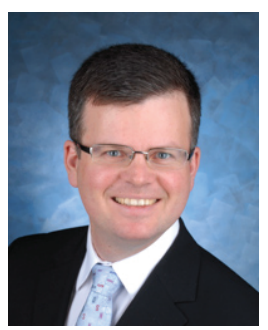

Markus Mueck oversees Intels technology development, standardization and partnerships in the field of spectrum sharing. In this capacity, he has contributed to standardization and regulatory efforts on various topics including spectrum sharing within numerous industry standards bodies, including ETSI, 3GPP, IEEE, the Wireless Innovation Forum and CEPT. Dr. Mueck is an adjunct professor of engineering at Macquarie University, Sydney, Australia, he acts as ETSI Board Member supported by INTEL and as general Chairman of ETSI RRS Technical Body (Software Radio and Cognitive Radio Standardization). He has earned engineering degrees from the University of Stuttgart, Germany and the Ecole Nationale Supérieure des Télécommunications (ENST) in Paris, France, as well as a doctorate degree of ENST in Communications.

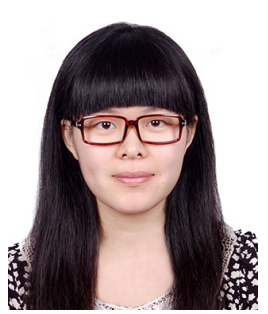

Ying He received the B.E. in Telecommunications Engineering degree in Beijing University of Posts and Telecommunication, Beijing, China in 2009. She developed the physical layer design for the TD-LTE baseband chip project in the Institute of Computing Technologies, Chinese Academy of Sciences, Beijing, China, from 2009 to 2013. She is currently a Ph.D. student in the Department of Engineering, Macquarie University, Sydney, Australia. Her research interests are spectrum sharing and resource allocation in next generation wireless networks. 